\title{
Propuesta Metodológica para el Rediseño de una Red Meteorológica en un Sector de la Región Andina Colombiana
}

\section{Proposed Methodology for the Redesign of Meteorological Network in a Sector of the Colombian Andean Region}

\author{
Víctor Leonardo López Jiménez \\ Ingeniería Ambiental y Sanitaria, Universidad de La Salle, Bogotá, Colombia, \\ vlopez@unisalle.edu.co
}

Recibido: 14 /07/2013 • Aprobado: 02/09/2013

\section{RESUMEN}

En sectores de Colombia, como los departamentos andinos, acompañados de una gran diversidad topográfica y ecosistémica, es importante contar con una red de estaciones, bien distribuida, para el monitoreo de las condiciones atmosféricas y lo suficientemente densa como para poder asegurar series de datos consistentes y así alimentar y fortalecer las estadísticas que hablan sobre el régimen climático de la región. La información, una vez depurada, es requerida por estudios e investigaciones sobre la ocurrencia de fenómenos meteorológicos, con el fin de mejorar la planificación y ejecución de programas en sectores como: el agrícola, ganadero, energético, transporte, salud, comercio e infraestructura; el seguimiento al comportamiento de las variables meteorológicas permite, además, fortalecer y/o crear nuevos programas orientados a la gestión del riesgo y a la planificación ambiental del territorio. En la propuesta metodológica para el rediseño de una red se tomó como área piloto el departamento de Cundinamarca, en el centro de la región Andina colombiana, no solo por sus características físico-geográficas y ambientales, sino porque en este sector se encuentran instaladas actualmente un importante número de estaciones meteorológicas. Es de anotar que, a pesar de la cantidad de información climatológica y ambiental, se ha detectado que la distribución, infraestructura y operación de la red no es la ideal. La metodología utilizada en la propuesta de rediseño de la red, se basa en las teorías expuestas por Gandín y Kagan (expertos en el diseño de redes); en los manuales, normas y protocolos publicados por la Organización Meteorológica Mundial (OMM) y en las experiencias y desarrollos alcanzados por el Ideam (entidad a cargo la meteorología en Colombia).

Palabras clave: condiciones climáticas, distribución espacial, operación de la red, red meteorológica. 


\section{AbStRact}

In areas of Colombia, such as the Andean departments, accompanied by a large topographic and ecosystem diversity, it is important to have a network of stations to monitor the weather conditions which is well distributed, dense enough to ensure consistent data series for nourish and strengthen statistics that talk about the climate system of the region. The refined information is required for studies and research about the occurrence of weather phenomena, in order to improve the planning and implementation of programs in sectors such as agriculture, animal husbandry, energy, transport, health, trade and infrastructure; monitoring the behavior of the meteorological variables also allows strengthen and / or create new oriented risk management and environmental territorial planning programs. In the proposed methodology for network redesign pilot area the department of Cundinamarca in central Colombia's Andean region was taken not only for their physical-geographical and environmental characteristics, but because in this area there are currently installed an important number of weather stations. Should be noted that, despite the amount of weather and environmental information, it was detected that the distribution infrastructure and network operation is not ideal. The methodology used in the proposed redesign of the network is based on the theories presented by Gandin and Kagan (experts in network design); also in manuals, regulations and published by the World Meteorological Organization (WMO) protocols and experiences and developments achieved by the IDEAM (entity in charge of meteorology at Colombia).

Keywords: climatic conditions, meteorological network, spatial distribution, network operation.

\section{Generalidades Sobre Redes de ObSERVACIÓN Meteorológica}

El comportamiento del clima condiciona, en buena medida, la mayoría de las actividades que el ser humano realiza, más aún cuando estas tienen que ver con la planificación y ejecución de programas relacionados con el desarrollo de una región y el bienestar de su población. Es necesario conocer la distribución mensual de las lluvias y la temperatura, por ejemplo, cuando se programa una cosecha o se construye una vía, o cuando se quiere prever la ocurrencia de eventos adversos como desbordamientos, inundaciones, deslizamientos, heladas o sequías. Esto solo es posible cuando se cuenta con una consistente serie de datos, lo que se logra con una red meteorológica que permita obtener datos consistentes, representativos y veraces.

\section{A. Redes meteorológicas.}

[1] define una red meteorológica como "el conjunto de estaciones, convenientemente distribui- das, en las que se observan, miden y/ o registran las diferentes variables, fenómenos y elementos atmosféricos que son necesarios en el conocimiento y la determinación del estado del tiempo y el clima de una región, para su posterior aplicación a diversos usos y objetivos". Tomando como referencia los criterios recomendados por [1], las condiciones fisiográficas y de diversidad climática del territorio colombiano, la optimización del uso de la red meteorológica para fines múltiples y la necesidad de contar con información consistente en el campo de la investigación, se hace necesario planificar el redimensionamiento de la red existente en el país.

\section{B. Estaciones meteorológicas.}

[2] define como estación meteorológica "el lugar donde se hacen las observaciones y mediciones puntuales de los diferentes parámetros 
meteorológicos, usando instrumentos apropiados, con el fin de establecer el comportamiento atmosférico de las zonas de un territorio". Las estaciones meteorológicas son clasificadas de acuerdo con las normas técnicas de la OMM, teniendo en cuenta los fines para los que son instaladas, como: pluviométricas (PM), pluviográficas (PG), climatológicas principales y ordinarias (CP y $\mathrm{CO}$ ), agrometeorológicas (AM), sinópticas principales y secundarias (SP y SS) y meteorológicas especiales (ME).

La densidad requerida para la observación de los datos meteorológicos está directamente relacionada con las escalas temporales y espaciales de los fenómenos atmosféricos que han de analizarse [1] La [3] clasifica las escalas de los fenómenos atmosféricos de acuerdo con su alcance horizontal en: pequeña escala, menos de 100 $\mathrm{Km}$, por ejemplo, tormentas eléctricas, vientos locales; meso-escala, entre 100 y $1000 \mathrm{Km}$, por ejemplo, frentes y formación de núcleos nubosos; gran escala, entre 1000 y $5000 \mathrm{Km}$, por ejemplo, depresiones, ondas tropicales y escala planetaria y más de $5000 \mathrm{Km}$, por ejemplo, aparición de ondas largas en la alta troposfera.

\section{Emplazamiento y exposición del instru- mental.}

La ubicación de la estación es de suma importancia y los errores de representatividad pueden ser mucho mayores que los previstos cuando se trata de estaciones aisladas. Estaciones ubicadas en zonas de montaña, como es el caso de muchas de las que conforman la red que se opera en Cundinamarca, muy probablemente no sean representativas de fenómenos a macro y mesoescala; sin embargo, una buena serie de datos y la homogeneidad de los mismos pueden ser de gran utilidad para los usuarios. De acuerdo con [1], los instrumentos deben ser instalados en un lugar geográfico delimitado y en un espacio aproximado de 10 por 7 o 14 metros, según su categoría; la estación debe estar sobre terreno llano y cubierto de grama o hierba baja. En lo posible no debe haber laderas próximas y no debe ubicarse en una hondonada. Además, debe estar alejada de árboles, edificios y otros obstáculos. La distancia del obstáculo más próximo al pluviómetro debe duplicar y, preferiblemente, cuadruplicar la altura del obstáculo.

\section{Cambios en el empleo y ubicación de ins- trumentos.}

Es muy posible que el emplazamiento de la estación deba ser cambiado con el tiempo debido al crecimiento de árboles, construcción de edificios y trazado de vías, entre otros. La reubicación de la estación debe hacerse tratando de que se reduzcan al mínimo los posibles efectos del cambio. Cuando se efectúan cambios en el instrumental, estos podrían introducir cambios en la medición, razón por la cual las observaciones realizadas con nuevos instrumentos deben compararse durante un período no inferior a un año antes de abandonar el anterior sistema. De acuerdo con la OMM [3], los instrumentos, así como la infraestructura de la estación observación (automática o convencional), deben recibir mantenimiento regular para evitar el deterioro del instrumental y de la calidad de las observaciones. Los programas de mantenimiento preventivo de las estaciones comprenden cuidados físicos como el corte de la hierba y la limpieza de superficies e instrumentos.

\section{E. Tratamiento de la información.}

La información una vez recolectada debe ser verificada, ejercicio mediante el cual se verá claramente la incidencia de factores meteorológicos como la orografía y se podrán detectar errores nacidos del mal funcionamiento del instrumental y/o de la falta de destreza de los observadores. Este proceso es importante ya que de la pureza de los datos dependen los resultados de los estudios e investigaciones en los que estos sean utilizados [4].

\section{F. Descripción del área de estudio.}

Cundinamarca posee 116 municipios, distribuidos en 15 provincias: Almeidas, Alto Magdalena, Bajo Magdalena, Gualivá, Guavio, Magdalena Centro, Medina, Oriente, Rionegro, Sabana Centro, Sabana Occidente, Soacha, Sumapaz, Tequendama y Ubaté. Latitudinalmente se extiende 
entre los $05^{\circ} 50^{\prime}$ y los $03^{\circ} 40^{\prime}$ al Norte del Ecuador y longitudinalmente entre los $74^{\circ} 54^{\prime}$ y los $73^{\circ} 28^{\prime}$ al Oeste de Greenwich [5].

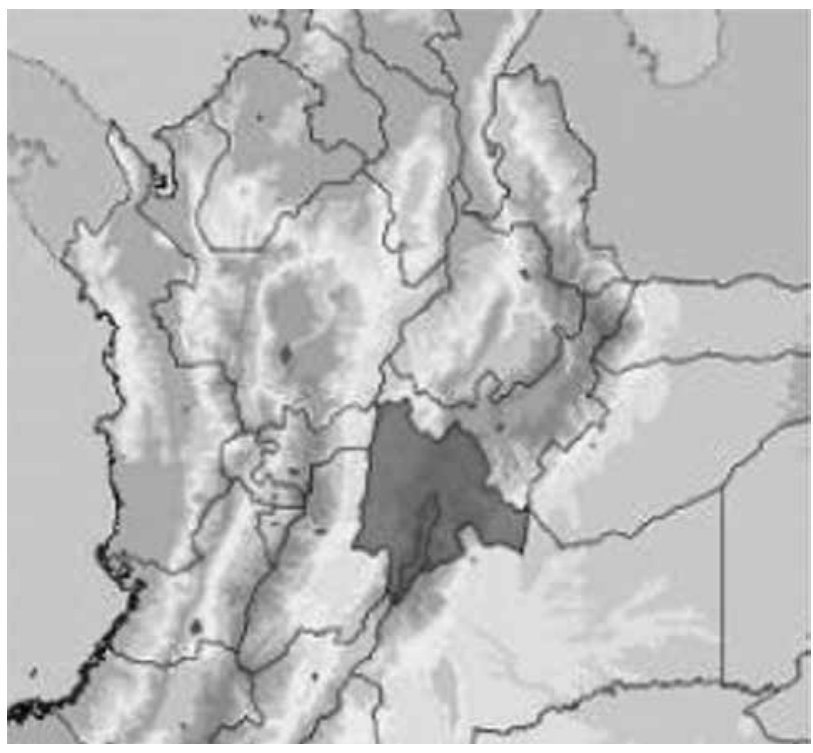

Fuente: http://www.ecoturismo-colombia.com/

Fig. 1 Ubicación del área de estudio.

\section{G. Fisiografía.}

La mayor parte del departamento está localizada sobre la vertiente occidental de la cordillera Oriental, incluyendo la Sabana de Bogotá. En el extremo sur, sobre la cordillera Oriental, se encuentra ubicado el Páramo de Sumapaz, con elevaciones de hasta 4.250 m.s.n.m.; en la parte intermedia de esta cordillera se localiza el altiplano de la Sabana de Bogotá y hacia el norte y noreste se encuentran los valles de Ubaté y Simijaca, en una elevación promedio de 2.600 m.s.n.m.

Ambos costados de la cordillera Oriental tienen, además, zonas de montaña: el flanco occidental drena hacia el valle geográfico del río Magdalena el cual tiene una elevación promedio de 1.500 metros; el flanco oriental tiene, en cambio, un paisaje quebrado con elevaciones que en promedio alcanzan 3.000 m.s.n.m. Para tener una idea de la distribución porcentual de los diferentes rangos de elevación, en la tabla 1 se presentan, además de estos, las áreas y sus correspondientes porcentajes.
TABLA I

Distribución Hipsométrica Porcentual

\begin{tabular}{|c|c|c|}
\hline $\begin{array}{c}\text { Rango de elevación } \\
(\mathbf{m s n m})\end{array}$ & Área $\left(\mathrm{Km}^{2}\right)$ & $\%$ \\
\hline $4250-4000$ & 17.99 & 0.1 \\
\hline $4000-3500$ & 880.24 & 0.4 \\
\hline $3500-3000$ & 2135.86 & 11.4 \\
\hline $3000-2500$ & 5657.60 & 30.3 \\
\hline $2500-2000$ & 1260.89 & 6.7 \\
\hline $2000-1500$ & 1491.14 & 8.0 \\
\hline $1500-1000$ & 2606.16 & 13.9 \\
\hline $1000-500$ & 2474.26 & 13.2 \\
\hline $500-200$ & 2161.77 & 11.6 \\
\hline TOTAL & 18685.91 & 100.0 \\
\hline
\end{tabular}

Fuente: Atlas Ambiental CAR (2002)

Se concluye que, en el territorio cundinamarqués existen cuatro paisajes fisiográficos claramente definidos: el valle del río Magdalena, las formas de montaña en la vertiente occidental de la cordillera Oriental, las formas de alta montaña en la vertiente oriental de la cordillera Oriental y la planicie fluviolacustre de los ríos Bogotá y Ubaté - Suárez.

\section{H. Hidrografía.}

El altiplano Cundiboyacense sirve de divorcio de aguas hacia el Occidente al sistema fluvial del río Magdalena (se extiende altitudinalmente desde los 2.000 hasta los 1.000 m.s.n.m). A este sistema fluvial del Magdalena que ocupa el $94 \%$ del territorio cundinamarqués confluyen los ríos: Sumapaz, Bogotá, Seco, Negro, Minero, Ubaté y Suárez. Los ríos que drenan hacia el Oriente y que en los Llanos Orientales hacen confluencia con los ríos Meta y Guaviare, son: el río Gachetá, el río Blanco y el río Machetá. 
Respecto de la morfometría de las cuencas hidrográficas, la cuenca del río Bogotá es la que ocupa la mayor porción de área, seguida de las cuencas del río Negro, el río Sumapaz y el río Magdalena, en su orden.

\section{Climatología.}

El área tomada como piloto para el estudio está distribuida en elevaciones que van desde los 150 hasta los 4.150 m.s.n.m. por lo que es posible encontrar sectores del territorio en el piso térmico cálido, como es el caso de las riberas del río Magdalena, al igual que lugares con temperaturas muy bajas como el Páramo de Sumapaz. La rica distribución climática de la región permite encontrar lugares con diferentes niveles de humedad; esta variedad va desde las zonas secas en el sector del municipio de Ráquira (Boyacá) hasta las zonas húmedas localizadas en las cuencas de los ríos Blanco y Negro.

Como resultado de la zonificación climática, de acuerdo con la clasificación propuesta por [6], se pueden diferenciar en la región varios climas. En la parte norte de la cuenca del río Magdalena y la parte baja de la cuenca del río Negro, aguas abajo del municipio de Caparrapí, se identifica un clima tropical lluvioso de selva, que se caracteriza por un período lluvioso casi constante a lo largo del año, con la presencia de dos cortos períodos secos. En estos sitios se presenta una cobertura vegetal densa y uniforme. En el sector occidental, en la parte media de la cuenca del río Negro, gran parte de la cuenca del río Magdalena y las partes bajas de los ríos Bogotá y Sumapaz, se encuentra un clima de tipo tropical lluvioso de sabana. Este clima se caracteriza por presentar una temporada seca bien definida y la precipitación media anual es inferior a los $2.500 \mathrm{~mm}$.

Existe un tercer tipo de clima, presente en gran parte de la franja media del departamento de Cundinamarca identificado como templado húmedo de verano seco. Este clima es evidente a lo largo de las sabanas de los ríos Bogotá y Suárez, por debajo de los $2.800 \mathrm{msnm}$, en la parte alta de la cuenca de los ríos Negro y Minero y en la parte media de la cuenca del río Sumapaz. En zonas de altitudes mayores a los $2.800 \mathrm{msnm}$ el clima es frío, de alta montaña, con temperaturas promedio por debajo de los $10^{\circ} \mathrm{C}$. Es posible, además, encontrar otros tipos de clima como el tropical lluvioso de bosque y el templado húmedo con lluvias todo el año, presentes en diferentes áreas a lo largo de Cundinamarca (territorio considerado para el estudio). La variedad de climas relacionada, como ya se anotó, con la diversidad orográfica obliga a que la información meteorológica deba ser tomada desde una nutrida y bien distribuida red de estaciones [7].

\section{Análisis Metodológico}

Teniendo en cuenta que el rediseño de la red meteorológica para un área de estudio debe dar como resultado alternativas de distribución y densidad de las estaciones que la conforman, se realizó la búsqueda de una metodología que se ajustara a las condiciones del entorno físico, los aspectos técnicos, los procesos socio-económicos y los elementos culturales de la región. Para esto se efectuó la revisión de varios de los métodos expuestos por expertos en redes, algunos de ellos publicados por la OMM [1]. También se tuvieron en cuenta propuestas consignadas en documentos del Ideam [8].

\section{A. Método de Gandín.}

En [9] y [10] expone que para el diseño de redes de estaciones meteorológicas se deben tener en cuenta los parámetros climatológicos por ser observados ya que la densidad de la distribución de estaciones dependerá de estos [10]. En la nota se hace claridad sobre la composición de una red meteorológica básica, la importancia de las estaciones de referencia y el significado de las estaciones especiales o transitorias. Las estaciones de una red básica solo podrían dejar de funcionar en casos excepcionales; por ejemplo, como resultado de la construcción de obras civiles, momento en el que la estación dejaría de ser representativa. En este caso, se debe buscar la manera de reubicarla en el 
lugar más cercano posible, teniendo cuidado de que cumpla con las especificaciones para su instalación. Para evitar estos inconvenientes, la selección de puntos de observación debe hacerse considerando que las estaciones de una red básica deben, en lo posible, no ser trasladadas de sitio. De acuerdo con la clasificación adoptada por la OMM, como se muestra en la tabla 2, existen tres grupos de redes de estaciones para la toma de datos y el desarrollo de observaciones meteorológicas.

TABLA II

Distribución de Estaciones en una Red Básica.

\begin{tabular}{|c|c|c|c|}
\hline Grupo & $\begin{array}{l}\text { Tipo de } \\
\text { estación }\end{array}$ & $\begin{array}{l}\text { Distancia } \\
(\mathrm{Km})\end{array}$ & Radio de acción promedio (Km) \\
\hline $1-R$ & Referencia. & De 300 a 400 & $\begin{array}{l}\text { Entre } 150 \text { y } 200 \\
\text { * Una por cada } 405 \text { del primer grupo }\end{array}$ \\
\hline 1 & $S P-C P-A M$ & De 150 a 200 & $\begin{array}{l}\text { Entre } 75 \text { y } 100 \\
\text { *Aprox. una por cada } 10 \text { estaciones del } 2 \text { grupo }\end{array}$ \\
\hline 2 & $\mathrm{CO}-\mathrm{ME}$ & De 50 a 60 & $\begin{array}{l}\text { Entre } 25 \text { y } 30 \\
{ }^{*} \text { Aprox. } 10 \text { estaciones por una del primer grupo }\end{array}$ \\
\hline 3 & $P M-P G$ & De 25 a 30 & $\begin{array}{l}\text { Entre } 12 \text { y } 15 \\
\text { * La mitad de la distancia en áreas montañosas }\end{array}$ \\
\hline
\end{tabular}

* Sugerencia técnica OMM.

Fuente: Nota téc. No. 111 -OMM. Adaptado López. 2008
El primer grupo de redes de estaciones se recomienda que esté especialmente adaptado a las condiciones físicas y geográficas de la región y que la distancia entre ellas sea de 150 a $200 \mathrm{Km}$. De este primer grupo de estaciones es importante seleccionar un número de estaciones de referencia las cuales deben poseer extensas y sólidas series de datos ya que sirven, especialmente, para dos propósitos: la corrección y la complementación de datos de estaciones vecinas y para estudios de variabilidad y cambio climático. Las estaciones de referencia deben permanecer largos periodos de tiempo sin tener que ser cambiadas de sitio.

[9] sugiere que la distancia entre estaciones de referencia sea de entre 300 y 400 Km; además, sería deseable que por cada 4 o 5 estaciones del primer grupo exista una de referencia.

En las estaciones del segundo grupo se realiza un volumen de observaciones menor que en las del primer grupo. Se considera que los puntos de monitoreo de segundo orden deben distar unos de otros entre $50 \mathrm{Km}$ y $60 \mathrm{Km}$; esto quiere decir que aproximadamente de cada 10 estaciones del segundo grupo habría una del primer grupo. A este grupo pertenecerían las estaciones climatológicas ordinarias (CO) y la red de estaciones para fines especiales (ME)

En un tercer grupo se incluyen las estaciones pluviométricas (PM) y pluviográficas (PG), en las cuales solamente se hace la observación de fenómenos atmosféricos relacionados con la precipitación (lluvia y/o nieve). La distancia racional entre estas estaciones es de 25 a $30 \mathrm{Km}$ en terreno plano y de la mitad de esta distancia en áreas montañosas. Las consideraciones sobre la distancia entre estaciones de una red se aplican, en primera instancia, a terrenos de condición plana. Para áreas de topografía quebrada o condiciones montañosas, varía mucho esta densidad, teniendo en cuenta el comportamiento espacio-temporal 
de los diferentes parámetros climáticos. Es importante considerar que la red básica (estaciones de primer, segundo y tercer orden), eventualmente, puede estar apoyada por una red de estaciones especiales que funcionarían por cortos espacios de tiempo según las necesidades existentes.

\section{B. Método de correlación espacial de Kagan.}

En conexión con los intereses enseñados por la Comisión de Climatología de la OMM, en el problema de la distribución racional de redes, la oficina central del Observatorio de Geofísica ha estado recopilando experiencias sobre el asunto. A continuación se hace una breve descripción de la metodología expuesta por R. I. Kagan expuesta en [11].

Tener en mente el interés del proyecto es más importante que la figura de los puntos que, individualmente, conforman la red. El resultado de los cálculos hechos por Kagan muestra que la función de correlación de la precipitación es, sustancialmente, diferente en regiones fisiográficamente diferentes. La base del método de Kagan es la función de correlación r(d), la cual es dependiente del espaciamiento entre estaciones y su forma depende de las características del área considerada y del tipo de precipitación, que puede ser expresado matemáticamente de la siguiente manera.

$$
\rho(d)=\rho(o) \cdot e^{-\frac{d}{d_{o}}}
$$

Donde:

$d$ - Distancia entre estaciones.

r(o - Coeficiente de correlación cruzada para una distancia $d=0$.

$d_{0}$. Radio de correlación, o distancia a la cual el coeficiente es $r(0) / e$.

Teóricamente $r(0)$ debería ser igual a la unidad; sin embargo, es menor debido a los errores aleatorios en la medición de la precipitación y a la diferencia microclimática que podría presentarse en el área de estudio. [12] estima la varianza de dichos errores de acuerdo con la siguiente expresión:

$$
\sigma_{1}^{2}=[1-\rho(o)] \sigma_{P}^{2}
$$

Donde:

$s_{\mathrm{p}}{ }^{2-}$ Varianza de la precipitación puntual

Los parámetros $r(0)$ y $d_{0}$ los usa Kagan para determinar la bondad de la red meteorológica siguiendo los dos criterios siguientes:

1) Evaluación de la predicción de la Iluvia promedio. La varianza del error de predicción media sobre un área unitaria, "a", con una estación central se define como la suma del error de medición dado y la variación espacial. De tal modo, que la varianza para el área total "A" con " $n$ " estaciones uniformemente distribuidas está dada por la siguiente expresión:

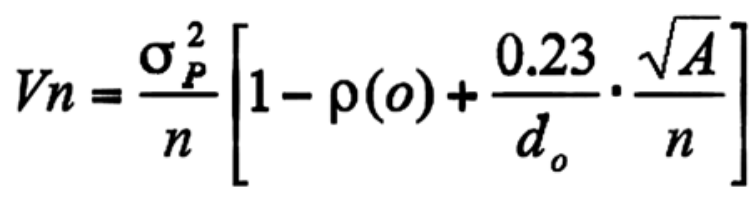

El error cuadrático medio relativo $\left(Z_{1}\right)$ es, entonces:

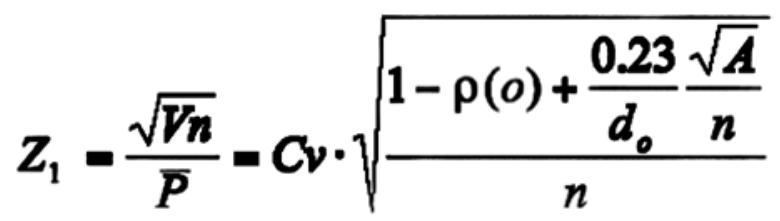

Donde:

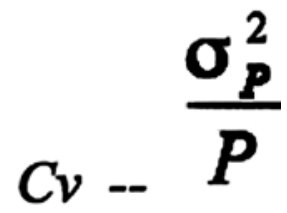

$P$ - Precipitación media sobre el área A.

Conocidos $r(0)$ y $d_{o}$, la ecuación (4) proporciona el valor de $\mathrm{n}$ (número de estaciones) para un error (Z1) fijo y viceversa. Si se adopta una distribución cuadrangular, el espaciamiento entre estaciones será: 


$$
L=\sqrt{\frac{A}{n}}
$$

Donde:

L- Espaciamiento entre estaciones.

Pero, cuando el área posee una configuración compleja se recomienda que la distribución sea triangular, de modo que el espaciamiento está dado por:

$$
L=1.07 \sqrt{\frac{A}{n}}
$$

2) Evaluación de la predicción de la interpolación espacial. El error relativo asociado con una interpolación lineal entre dos puntos e interpolación hacia el centro de un cuadrángulo o triángulo, donde ocurre el máximo error, es dado por [19] para una distribución triangular por la siguiente expresión:

$$
Z_{3}=C v \cdot \sqrt{\frac{1}{3}[1-\rho(0)]+0.52 \cdot \frac{\rho(0)}{d_{o}} \frac{\sqrt{A}}{n}}
$$

Donde: $C v-\frac{\sigma_{P}^{2}}{\bar{P}}$

La evaluación de $Z_{1}$ y $Z_{3}$ requiere del conocimiento de $r(0)$ y $d_{0}$, los cuales pueden ser evaluados de la función $r(d)$ deducida de los datos observados. Además, al aplicar el método se debe tener en cuenta que el valor de precisión $Z$ máximo por considerar es del $10 \%$. Sin embargo, esto se constituye en un procedimiento arbitrario, por lo que también es razonable el análisis de un descenso no significativo del valor de $Z$ con el incremento del número de estaciones, aunque este se encuentre por arriba del $10 \%$.

\section{Método de red mínima - OMM.}

De acuerdo con la OMM [13], el valor de los datos derivados de una red hidrometeorológica depende de la utilización que, finalmente, se les dé. Una red básica debiera suministrar, entonces, un nivel de información suficiente para ser aplicada en estudios sobre el comportamiento del clima en un lugar cualquiera dentro de la región en que opera la red [14], [18]. Para poder conseguir esto deben abarcarse por lo menos tres criterios:

- Disponer de un sistema para la transferencia de información desde los lugares donde se recoge hasta cualquier otro lugar en el área.

- Existir un medio para estimar la cantidad de información tomada de las estaciones para evitar la incertidumbre

- Disponer del mayor número de datos antes de tomar las decisiones finales.

En la nota técnica 168 , refiriéndose a los recursos hídricos [7]; la [13] muestra que la primera etapa en la creación de una red básica eficiente es el establecimiento de una red mínima. Esta red debería estar compuesta por el menor número de estaciones que la experiencia colectiva de los servicios hidrometeorológicos haya indicado como necesarias para la planificación de los recursos. Es recomendable que la red mínima se desarrolle tan rápido como sea posible, incorporando las estaciones existentes de acuerdo con el tipo de información que estas suministren. De esta manera, la red mínima se convierte en la estructura básica de la red en expansión para atender futuras necesidades en ámbitos específicos. Esta red mínima tiene asociada una densidad mínima, que para el caso de la red climatológica se encuentra clasificada de acuerdo con el tipo de datos que se recopilan.

\section{1) Densidad de estaciones para una red mínima.} Se pretende que el concepto de densidad de red sirva como orientación general en caso de carecer de una guía específica; estas densidades de 
diseño tienden a tener en cuenta las condiciones socioeconómicas y físico-climáticas reales. Las zonas poco pobladas tienen, en general, climas extremos; se trata de altas montañas, regiones áridas, polares o de bosques tropicales. En el otro extremo, las zonas urbanas densamente pobladas requieren de una red pluviométrica muy densa tanto para el estudio espacio-temporal como para el diseño, gestión y control en tiempo real. Para el caso en la Guía se definen seis tipos de regiones fisiográficas: zonas costeras, zonas montañosas, llanuras interiores, zonas escarpadas ondulantes, islas pequeñas (con superficies inferiores a $500 \mathrm{Km}^{2}$ ) y zonas polares y áridas.

2) Densidades para estaciones climatológicas. Se considera que en una estación climatológica que hace parte de una red básica se miden los parámetros, precipitación, evaporación, humedad, temperaturas y vientos. Los dos primeros son fundamentales en estaciones instaladas con fines hidrológicos.

3) Estaciones pluviométricas. En la tabla 3 se presentan los valores correspondientes a la densidad mínima de estaciones pluviométricas. Estos valores no se aplican a grandes desiertos ni extensiones considerables de hielo y se debe tener en cuenta que siguen ciertos principios de instalación y uso, con el fin de atender las necesidades más inmediatas. Los pluviómetros deben estar distribuidos de la manera más uniforme posible, teniendo en cuenta la necesidad de información y el personal de observadores disponible [7].

TABLA III

Densidad Mínima Recomendada Omm, Para Estaciones Pluviométricas

\begin{tabular}{l|c|c|}
\multirow{2}{*}{\multicolumn{1}{c|}{ UNIDAD FISIOGRÁFICA }} & \multicolumn{2}{|c}{$\begin{array}{c}\text { DENSIDAD MÍNIMA POR ESTACIÓN } \\
\text { (Superficie en Km² por estación) }\end{array}$} \\
\cline { 2 - 3 } Zonas costeras & Sin registro (PM) & Con registro (PG) \\
\hline Zonas montañosas & 900 & 9.000 \\
\hline Llanuras interiores & 250 & 2.500 \\
\hline Zonas escarpadas /ondulantes & 575 & 5.750 \\
\hline Pequeñas islas & 575 & 5.750 \\
\hline Zonas urbanas & 25 & 250 \\
\hline Zonas polares y áridas & & 10 a 20 \\
\hline
\end{tabular}

Fuente: Guía de prácticas hidrológicas, adquisición y proceso de datos, OMM - No. 168

En regiones montañosas, debe darse una atención especial a la distribución vertical tratando de cubrir al máximo las franjas hipsométricas y utilizando pluviómetros totalizadores para medir las precipitaciones a grandes altitudes.

4) Estaciones de evaporación. Una estación de evaporación consiste en un tanque de diseño convencional estándar, donde se toman los datos diarios de evaporación. En ellas se toma también la temperatura del aire, la humedad, el punto de rocío y el comportamiento del viento. En la tabla 4 figuran las recomendaciones para una red mínima de estaciones de evaporación en zonas de fisiografía uniforme [7]. 
TABLA IV

Densidad Mínima Recomendada para EstaCIONES DE EVAPORACIÓN

\begin{tabular}{|l|c|}
\hline \multicolumn{1}{|c|}{$\begin{array}{c}\text { UNIDAD } \\
\text { FISIOGRAFICA }\end{array}$} & $\begin{array}{c}\text { DENSIDAD MÍNIMA } \\
\text { (Superficie en Km² } \\
\text { por estación) }\end{array}$ \\
\hline Zonas costeras & 50.000 \\
\hline Zonas montañosas & 50.000 \\
\hline Llanuras interiores & 50.000 \\
\hline Zonas escarpadas / \\
ondulantes
\end{tabular}

Fuente. Nota técnica 168 - OMM

La evaporación desempeña un papel importante en estudios a largo plazo del régimen hídrico de ríos, lagos y embalses, así como en la explotación del recurso agua. En todos los casos, el número y la distribución de estaciones de evaporación están determinados con base en la configuración y área de los cuerpos de agua y por la región climática en que se encuentran.

\section{5) Criterios de la "Red de Referencia} Preliminar". El objetivo que planteó [8], fue poder determinar un número inicial de estaciones (RRP) que representara las diferentes regiones naturales del país. Estas estaciones se seleccionaron de acuerdo con un conjunto de criterios relacionados con su elevación, longitud y calidad de las series disponibles; con su representatividad geográfica y climatológica y con la calidad del instrumental utilizado y la disponibilidad de los datos. El trabajo se orientó a la precipitación, teniendo en cuenta que es el parámetro de mayor variabilidad y, en consecuencia, los métodos desarrollados para su análisis estadístico son válidos para series con mayor estabilidad en la media y con menor varianza.
Los criterios establecidos para la determinación de la red básica de referencia fueron:

- La densidad por franjas hipsométricas, cada 1000 metros de elevación sobre el nivel medio del mar y sobre los altiplanos.

- La distribución de las estaciones a barlovento y sotavento de las cordilleras y en los valles de los grandes ríos.

- La extensión y validez de las series de datos.

- La calidad de la información suministrada por las estaciones.

- El tipo de estación y el estado y la calidad del instrumental.

- Número de veces que la información se utilizó en estudios e investigaciones por su calidad.

- El cumplimiento de las normas de instalación (área despejada, plana, baja cobertura, etc.).

- Cumplimiento en horarios de observación y calidad en la transcripción de datos.

Para descartar estaciones en áreas en las que se observó una mayor concentración se optó por establecer la calidad de las series de manera empírica, recurriendo al concepto técnico de la verificación de información hidrometeorológica. Se escogieron las estaciones de mayor período de registro y las de mejor calidad en la información mediante la aplicación de pruebas estadísticas. Cumplidas las distintas fases del proceso se propuso, para la medición de la lluvia en los departamentos de Colombia, una densidad de red básica según el método del Ideam, (tabla 5). 
TABLA V

Densidad Promedio de la Red por Departamento

\begin{tabular}{|l|c|}
\multicolumn{1}{c|}{ RANGO DE ÁREAS (en $\mathbf{K m}^{\mathbf{2}}$ ) } & Número de estaciones por $\mathbf{K m}^{\mathbf{2}}$ \\
\hline Dep. con áreas entre 40 y $10.000 \mathrm{Km}^{2}$ & 2,43 \\
\hline Dep. con áreas entre 10.000 y 30.000 & 1,08 \\
\hline Dep. con áreas entre 30.000 y 60.000 & 0,34 \\
\hline Dep. con áreas entre 60.000 y 90.000 & 0,24 \\
\hline Territorios con área mayor a 100.000 & 0,07 \\
\hline
\end{tabular}

Fuente: Estudio Preliminar IDEAM. 1996

Bajo ese concepto metodológico fueron sugeridas por el Ideam para el altiplano cundí-boyacense: para la red de Cundinamarca 1,40 estaciones de referencia por cada $1000 \mathrm{Km}^{2}$ y para el departamento de Boyacá 1,72 estaciones por cada 1000 $\mathrm{Km}^{2}$, datos muy cercanos a los propuestos en [7], para pluviómetros con registro (PG).

6) Consideraciones sobre una red mínima funcional. Se considera una red mínima funcional aquella cuya capacidad físico-técnica permite medir y/o registrar de manera consistente y continua las variaciones que presentan las distintas variables meteorológicas, así como la ocurrencia de fenómenos atmosféricos en un área determinada. Tomando como base los conceptos de red básica y red mínima expuestos en párrafos anteriores y considerando la diversidad de condiciones atmosféricas que pueden afectar un territorio en el tiempo, es posible plantear una distribución aproximada de las estaciones que deben conformar una red climatológica mínima y funcional. Los tipos de estaciones meteorológicas tenidas en cuenta en una red básica son: Sinópticas, Agro-meteorológicas, Climatológicas y Pluviométricas.

Considerando la nota técnica 111-OMM [9] y [18] así como normas y anotaciones incluidas en otras publicaciones, se construyó la tabla 6 , en la que aparecen los valores medios mínimos tomados para la distancia entre estaciones y como radio de acción (cobertura) de estas. En esta tabla no se consideran las estaciones sinópticas a las que se atribuyen funciones aeronáuticas, aunque en algunos casos presten servicio como climatológicas (al interior de los estudios). Es de anotar que estos valores los determina la OMM para territorios donde no hay presencia de accidentes orográficos que alteren el comportamiento de los elementos meteorológicos, razón por la cual se plantean ajustes que responden a la importancia de la orografía como factor climatológico.

TABLA VI

Valores Medios para Red Mínima Funcional-Omm

\begin{tabular}{l|c|c|}
\multicolumn{1}{|c|}{ TIPO } & Distancia media (Km) & Radio de acción (Km) \\
\hline Climatológica Principal (CP) y Agrometeorológica (AM) & 150 & 75 \\
\hline Climatológica Ordinaria (CO) & 50 & 25 \\
\hline Pluviométrica (PM - PG) & 25 & 13 \\
\hline
\end{tabular}

Fuente: nota técnica No. 111 - OMM, 1970 
De acuerdo con [15], "la combinación de la pendiente y la dirección de la vertiente definen el aspecto físico y climatológico de un lugar". Es claro entonces que, la orografía tiene una fuerte incidencia sobre el comportamiento de las variables meteorológicas. Considerando la diversidad orográfica de Cundinamarca, se puede concluir que la densidad de la red meteorológica debe estar por encima del triple de los mínimos sugeridos por la OMM [1] ya que estos fueron calculados, como ya se dijo, para terrenos llanos o sin afectación por accidentes orográficos.

7) Generalidades sobre la red meteorológica actual de Cundinamarca. Con el fin de monitorear de manera permanente las condiciones atmosféricas y cumplir con la demanda de información por parte de las instituciones y la sociedad en general para la programación de sus actividades, Colombia cuenta con una red de estaciones distribuidas a lo largo y ancho del territorio. El Ideam y la CAR (esta última en Cundinamarca), además de velar por la continua operación de las estaciones que conforman la red, se ocupan del acopio de los datos, con los que es alimentado el banco nacional de información.

\section{8) Distribución de las estaciones por categoría.} Las 265 estaciones que componen la red meteorológica (operadas por las dos instituciones consideradas en el estudio) corresponden a las categorías ya expuestas y están ubicadas en los diferentes municipios que conforman el departamento. En la tabla 7, se presenta la distribución de acuerdo con las entidades que se hacen cargo de la operación.

TABLA VII

Estaciones Activas de la Red Cundinamarca

\begin{tabular}{|c|c|c|c|}
\multicolumn{2}{c|}{ ESTACIONES CAR } & \multicolumn{2}{c|}{ ESTACIONES IDEAM } \\
\hline Categoría & Cantidad & Categoría & Cantidad \\
\hline CP & 26 & CP & 2 \\
\hline CO & 12 & CO & 21 \\
\hline PG & 42 & PG & 8 \\
\hline PM & 43 & PM & 57 \\
\hline AU & 19 & AU & 15 \\
\hline & & ME & 16 \\
\hline TOTAL & & AM & 1 \\
\hline
\end{tabular}

Fuente: elaboración propia

9) Consideraciones sobre la operación de la red. Un aspecto importante en la redimensión de una red meteorológica es determinar la calidad en la operación de la misma. Para esto es necesario hacer una revisión (verificación en lo posible) de la in- formación disponible obtenida de las distintas estaciones, considerando la extensión y continuidad de la serie; el comportamiento de los datos de acuerdo con un rango de valores establecido para cada uno de los parámetros, según la ubicación de la 
estación y la posible hora de observación (teniendo en cuenta promedios, notas y comentarios de los observadores e inspectores) [15] así como, el tipo de instrumental y su mantenimiento. Esta información contribuye con la selección de las estaciones que pueden ser tomadas como referencia en la red.

\section{REDISEÑO DE LA RED Meteorológica}

La definición de red meteorológica que presenta la OMM [1], incluye una "conveniente distribución", condición que no se cumple en Cundinamarca, región considerada para el estudio. Es importante anotar que, una de las razones por las que la red meteorológica que opera en el país no tiene una distribución y un cubrimiento que permita hacerla eficiente es la de no responder a un proceso de planificación conjunta.

\section{A. Distribución de la red actual en el área de estudio.}

Se pudo establecer que en Cundinamarca, como en la mayoría de los departamentos del país, se fue implementando la red meteorológicas de manera fraccionada e independiente de acuerdo con la necesidad de información, por lo que hoy en día es posible encontrar áreas en las que la densidad de las estaciones es muy grande y otras donde no hay puntos de observación suficientes para el monitoreo del comportamiento de los distintos parámetros climatológicos.

Como ya se mencionó en párrafos anteriores, la red meteorológica instalada en Cundinamarca está conformada por 265 estaciones, de las cuales más del $55 \%$ son pluviométricas o pluviográficas. Así, una de las razones por la que se escogió este departamento como piloto para plantear una metodología que permitiera llevar a cabo la redimensión de una red meteorológica, fue la falta de información sobre parámetros distintos a la lluvia. La falta de estaciones climatológicas (con datos y registros de variables distintas a la lluvia) sumado a la mala distribución de estas son las principales razones por las que no se puede garantizar el suministro de la información requerida por estudios e investigaciones que den cuenta sobre el comportamiento y la variabilidad de las condiciones climáticas.

\section{B. Criterios de diseño para la nueva red.}

Al decidir sobre la metodología con la que se llevaría a cabo el rediseño de la red meteorológica se tuvieron en cuenta las notas técnicas de la OMM [17] sobre el emplazamiento de estaciones y las características de una red mínima, las consideraciones expuestas en el marco de referencia (generalidades) y los conceptos utilizados por Gandin [10] y [12], en sus teorías de diseño de redes. Además de las teorías y métodos analizados, fueron tenidas en cuenta las características físicas de la zona de estudio y aspectos particulares de la red como:

- Número de estaciones, distribución y ubicación de las mismas.

- Categoría de las estaciones (parámetros observados).

- Características morfológicas del terreno (montaña, valle, etc.).

- Extensión y consistencia de la serie de datos.

- Calidad de la información (sondeo sobre calidad de los datos - revisión de series).

- Estado del instrumental según concepto técnico institucional.

- Operación de la red según bitácoras de observación, informes de inspección y verificación de información.

Partiendo del análisis de las estaciones que conforman la red (distribución y operación) y teniendo en cuenta las teorías y los criterios mencionados se llegó a lo que, finalmente, se consideró como la metodología por seguir en el rediseño de la red en Cundinamarca como área piloto escogida. 
De lo expresado por la OMM en las notas técnicas revisadas [16], puede decirse que una red meteorológica mínima y funcional debe tener la capacidad físico-técnica que permita medir y/o registrar de manera consistente y continua las variaciones de los elementos meteorológicos, así como la ocurrencia de fenómenos atmosféricos en un área determinada.

Las características topográficas del terreno sobre el que se encuentra operando la red estudiada, en su mayoría sobre el conjunto orográfico de la cordillera Oriental, con fuertes pendientes en algunos casos, determinaron el ajuste hecho a la metodología de Gandín la cual fue pensada más para latitudes medias y altas que para latitudes próximas al Ecuador [9].

Como resultado del análisis de estas teorías se consideró, como se muestra en la tabla 8 , que la densidad de las estaciones en la zona de estudio debe crecer entre 4 y 6 veces por encima de la que propone la OMM para terrenos llanos o con algunas ondulaciones.

TABLA VIII

Valores Medios de Cobertura Para una Red Mínima Auustada

\begin{tabular}{|c|c|c|c|c|c|}
\hline \multirow{2}{*}{ Grupo } & \multirow{2}{*}{ Tipo } & \multicolumn{2}{|c|}{$\begin{array}{c}\text { Terreno sin accidentes } \\
\text { orográficos - OMM }\end{array}$} & \multicolumn{2}{|c|}{$\begin{array}{c}\text { Terreno montañoso } \\
\text { Cundinamarca }\end{array}$} \\
\hline & & $\begin{array}{l}\text { Distanc. media } \\
(\mathrm{Km})\end{array}$ & $\begin{array}{l}\text { Radio de acción } \\
(\mathrm{Km})\end{array}$ & $\begin{array}{l}\text { Distanc. media } \\
(\mathrm{Km})\end{array}$ & $\begin{array}{c}\text { Radio de acción } \\
(\mathrm{Km})\end{array}$ \\
\hline 1 & $\begin{array}{l}\text { Climatológica Principal } \\
\text { y Agro-meteorológica } \\
\text { (CP - AM) }\end{array}$ & 150 & 75 & $20-30$ & $10-15$ \\
\hline 2 & $\begin{array}{l}\text { Climatológica } \\
\text { Ordinaria } \\
(\mathrm{CO}-\mathrm{AU})\end{array}$ & 50 & 25 & 12 & 6 \\
\hline & $\begin{array}{l}\text { Meteorológica } \\
\text { especial (ME) }\end{array}$ & 25 & 13 & 6 & 3 \\
\hline 3 & $\begin{array}{l}\text { Pluviométrica } \\
\text { (PM - PG) }\end{array}$ & 25 & 13 & 6 & 3 \\
\hline
\end{tabular}

Fuente. Nota técnica 111-OMM. Adaptado L. López, 2008

Es importante anotar que, en las estaciones del primer grupo (AM y CP), tanto la distancia entre ellas como su radio de acción, obedecen más al tipo de instrumental y al número de variables observadas que a la posibilidad de tener una mayor cobertura sobre el comportamiento de los parámetros. El hecho de tener instrumentos registradores en estas estaciones, permite monitorear el funcionamiento de estaciones del segundo y tercer grupo ubicadas a su alrededor, en un radio de hasta 30 kilómetros. De otro lado, aunque las estaciones sinópticas (SP y SS) no aparecen en la tabla, estas son consideradas como del primer grupo al establecer la cobertura de la red, teniendo en cuenta que en muchas ocasiones cumplen funciones climatológicas.

Aunque las estaciones automáticas (AU) por su volumen de información y posibilidad de constituirse en una muy buena fuente de información debieran ubicarse en el grupo 1 (como si se tratara de una $\mathrm{CP}$ ), para el caso de la región seleccionada en el presente estudio se consideran como del grupo 2 teniendo en cuenta que, la serie de datos en la mayoría de estas estaciones es muy corta y que la mayor parte de ellas no cuenta con un buen seguimiento y calibración debido a inconvenientes relacionados, en la mayoría de los 
casos, con el costo y la disponibilidad de asistencia técnica. Por esto, se sugiere que las estaciones automáticas se ubiquen dentro del radio de cobertura de estaciones o equipos convencionales de referencia, con lo que se garantiza (patrona) el seguimiento a los sensores que dan cuenta del comportamiento de los distintos parámetros.

Es muy importante recordar lo que recomienda la OMM en su nota técnica № 111 [9], acerca de que la creación de una red de referencia, deberá estar conformada por estaciones con una muy sólida y extensa serie de datos y un excelente instrumental; OMM [9] recomienda, además, que la estación tenga una buena operación, es decir, que cuente con observadores capacitados.

Se espera que las estaciones consideradas del primer grupo (SP, AM, CP), cumplan con buena parte de las condiciones citadas por la OMM, ya que son estas las llamadas a convertirse en la base de la red de referencia.

\section{Presentación y Análisis de Resultados}

Para conocer la realidad sobre la distribución y eficiencia de la red de estaciones meteorológicas operadas en Cundinamarca (área tomada como piloto) y la que sería la red esperada con el rediseño, se elaboraron una serie de mapas de ubicación y cobertura, aplicando las directrices de la OMM [1] y los conceptos que se plantearon en el estudio para el mejoramiento de la red. Los mapas elaborados fueron:

- Red de estaciones meteorológicas que son operadas actualmente.

- Cobertura de la red actual para el monitoreo de la precipitación.

- Cobertura de la red actual de estaciones climatológicas.
- Red propuesta para el monitoreo de la precipitación.

- Red propuesta para el monitoreo de los parámetros climatológicos.

Como resultado del análisis de estos mapas fue posible obtener una serie de consideraciones técnicas sobre el actual emplazamiento de las estaciones, su área de cobertura y el tipo de información meteorológica generada por estas.

\section{A. Análisis de la red meteorológica.}

En el ejercicio de análisis, se tomaron como base solamente las estaciones activas, sin importar la fecha de instalación ni la extensión de la serie.

\section{1). Red para el monitoreo de la precipitación.} Como parte de la red de monitoreo de la precipitación se consideraron todas las estaciones meteorológicas, convencionales y automáticas, que operan en los municipios cundinamarqueses, partiendo del hecho de que todas ellas poseen como mínimo un pluviómetro.

Como ya se anotó, se tuvieron en cuenta las 265 estaciones meteorológicas (operadas por el Ideam y la Car, solamente), de las cuales 246 permiten estudiar la distribución espacial y temporal de las lluvias en la zona de estudio. Es importante anotar que tan solo el $23 \%$ de las estaciones instaladas, con las que se monitorea el comportamiento de la lluvia, son AM, CO y CP; el $56,5 \%$, PM y PG; el $12.8 \%$, AU y el 1,4\%, SP (consideradas con funciones climatológicas). La red cuenta, además, con un $6 \%$ de estaciones ME. Al analizar los mapas, se pudo establecer que el número de estaciones y su distribución brinda poca posibilidad de registros para el estudio de la intensidad, frecuencia y duración de la lluvia, en especial al oeste y noroeste de la región.

2) Red climatológica. De lo anotado se deduce que, del total de estaciones operadas en el departamento, son muy pocas las que cuentan con equipos para el seguimiento continuo de parámetros como la temperatura,la humedad, 
la radiación y el viento. Tan solo se cuenta con 20 estaciones dotadas de todos los aparatos registradores en la jurisdicción: dos estaciones sinópticas, 28 climatológicas principales, una agrometeorológica y 15 automáticas; el resto corresponde a la red pluviométrica (PM-PG), a las estaciones $\mathrm{CO}$ (registro de lluvia y brillo solar) y a las ME, estas últimas instaladas para monitorear especialmente la ocurrencia de heladas en la Sabana de Bogotá. Los informes de las instituciones que operan la red dejan ver que en los últimos años se han venido instalando estaciones automáticas de última generación, las cuales tienen la ventaja de contar con transmisión de datos vía satelital, aspecto que se ha constituido en una fortaleza para alimentar el sistema de alertas tempranas y prevención de desastres, así como el proceso de pronóstico meteorológico.

Aunque pocas, algunas estaciones automáticas se encuentran ubicadas al interior de jardines meteorológicos convencionales lo que facilita la validación de la información generada por sus sensores. Como ya se comentó en otros apartes del estudio, los sensores con que cuentan este tipo de estaciones son muy delicados y propensos a daños ocasionados por aspectos como la concentración de contaminantes y/o partículas sólidas en suspensión (polen, polvo y sales), la acción de insectos y, en algunos casos, las condiciones climatológicas extremas. Por lo anterior, estos equipos deben ser monitoreados de manera permanente, actividad en la que se hace uso del instrumental convencional instalado en los jardines meteorológicos.

El que 28 de las estaciones que se encuentran en operación en el departamento sean climatológicas principales, consideradas como de primer orden, es una buena razón para pensar en la posibilidad de crear una red de referencia. Las actas de instalación muestran que, además de la calidad del instrumental, el emplazamiento de las estaciones climatológicas de tipo convencional es bueno, en general. Estas condiciones facilitan la generación de información valiosa que pudiera ser mejor aprovechada si se diera cumplimiento a los lineamientos entregados por la OMM en materia de observaciones meteorológicas.

La red cuenta con 34 estaciones automáticas (operadas por las dos instituciones citadas) para el seguimiento de las condiciones climáticas de la región; sin embargo, la mayoría de estas estaciones no se encuentran emplazadas en jardines meteorológicos convencionales ni en lugares próximos a estos, situación que dificulta los procedimientos de validación de la información registrada por los sensores automáticos. Hace falta que, de manera sistemática, se lleve a cabo la redistribución de la red de tal manera que mediante instrumentos convencionales se pueda validar la información registrada en las estaciones automáticas que operan en el departamento. Por esta razón, este tipo de estaciones (AU) han sido consideradas como de segundo orden en el estudio.

\section{B. Análisis sobre la distribución espacial de la red.}

Al analizar el conjunto de estaciones que conforman la red se observaron áreas donde no existe cobertura alguna o donde solamente es factible hacer el seguimiento de uno o dos parámetros meteorológicos.

A continuación se presentan algunas consideraciones producto del análisis de la distribución espacial de las estaciones que son operadas en el departamento:

La distribución espacial de la red meteorológica operada por el Ideam solo permite hacer el seguimiento de las condiciones climatológicas de una manera apenas aceptable. Se observa un cubrimiento deficitario en el occidente de Cundinamarca (cuenca del río Magdalena), en el norte y noroccidente (cuencas bajas de los ríos Seco y Negro) al igual que al sur de este departamento, en la región del Sumapaz. También es notoria la ausencia de puntos de observación al este y noreste de la jurisdicción, en los municipios de Saboyá, Manta, Tibirita y Guatavita, principalmente. 
De otro lado, la red operada por la Corporación brinda una buena cobertura en amplios sectores de las diferentes provincias de la jurisdicción y, por ende, en las cuencas que administra; sin embargo, se observa una baja densidad de estaciones en el occidente del departamento (cuenca del río Magdalena), al noroccidente de este (cuencas bajas de los ríos Seco y Negro) y en el sector de la cuenca alta del río Sumapaz.

La poca cantidad de estaciones meteorológicas instaladas por encima de los $2.500 \mathrm{msnm}$, ofrece una muy pobre cobertura de la franja de alta montaña del departamento, situación que dificulta el seguimiento de los distintos parámetros que identifican el clima y limitan el éxito de los estudios hidrometeorológicos en la parte alta de las cuencas.

Es importante anotar que las estaciones, al ser operadas por entidades distintas, en muchas ocasiones se encuentran emplazadas unas muy cerca de otras. El hecho de que las estaciones no sean operadas por una sola institución, no ha permitido orientar los esfuerzos hacia una distribución de la red que permita cubrir de manera integral el espacio geográfico en estudio.

\section{Operación de la red y calidad de informa- ción.}

Además de lo ya expuesto sobre la distribución de las estaciones que conforman la red, es importante atender las recomendaciones de la OMM sobre la operación de estas y la calidad de la información.

La operación de las estaciones meteorológicas presenta algunos inconvenientes, entre otros, la falta de compromiso de los observadores debido, muy probablemente, a la escasa remuneración; problemas relacionados con los horarios de observación al no cumplir con las normas estipuladas por la OMM; la disminución en cantidad y calidad del instrumental y la falta de programas de capacitación.

Para lograr un ajuste en la operación de la red y una mejora en la calidad de la información se debe partir de una supervisión técnica y juiciosa de las estaciones y del ejercicio del equipo de observadores. De igual manera, es importante adelantar una mejora al proceso de manejo de la información que incluya la revisión y verificación de los datos que llegan a los centros de acopio y procesamiento. Para lograr lo anterior se hace necesario fortalecer no solo el equipo de inspectores, sino el grupo de expertos en manejo y verificación de la información.

\section{Propuesta de Ajuste A LA RED}

De acuerdo con las recomendaciones de la OMM [7], una red (estaciones dotadas del instrumental para hacer seguimiento al comportamiento de los parámetros que determinan el clima de un espacio geográfico) debe ajustarse en función del tiempo o permanencia hasta que las condiciones climatológicas y las relaciones locales o regionales puedan establecerse. Por ello, se debe tener cuidado, no solo en la ubicación e instalación de las estaciones, sino también en asegurar la continua operatividad y el control sobre la confiabilidad y exactitud de los datos, registros y demás información que en estas se produce.

De igual manera, [1], [7] y [17] recomiendan establecer el seguimiento y evaluación de la red mediante programas que permitan evaluar el nivel de representatividad de las estaciones que la componen; en este sentido, es importante la aplicación de métodos que ayuden a establecer el grado de correlación entre las estaciones y averiguar si sus aproximaciones son acordes para mostrar la confiabilidad de la información tomada en los distintos puntos de observación.

En el caso de Cundinamarca, hay interés por parte de las dos principales instituciones que operan la red para revisar de manera minuciosa la distribución de las estaciones y optimizar el funcionamiento de estas. Se pudo establecer que la red meteorológica conjunta ha tenido un 
crecimiento sostenido y continuo, aunque no bien planificado; en la actualidad se encuentran en operación 265 estaciones, las cuales cubren entre el 60 y $65 \%$ del área geográfica.

Debido a que la red conjunta no está cumpliendo con la cobertura para hacer el seguimiento requerido al comportamiento del clima en la región, se hizo necesario estudiar la posibilidad de ajustar las distancias entre estaciones y reubicar algunas de ellas para mejorar su representatividad.

Con respecto al diseño y/o ajuste de una red meteorológica, [10], aunque no se refiere a las condiciones geográficas ni atmosféricas de las latitudes tropicales, establece grados de correlación y aceptabilidad entre las estaciones, tiempo de las series y áreas de representatividad. Además, expone que para el diseño de estas redes es muy importante tener en cuenta los parámetros climatológicos por ser observados ya que de este factor depende la densidad de estaciones con que debe contar la red. En la nota técnica también se hace claridad sobre la composición de una red meteorológica básica, la importancia de las estaciones de referencia y el significado de aquellas consideradas especiales o transitorias.

Con base en el documento de [10], en las demás teorías OMM (red mínima, red básica, red de referencia etc.) y en criterios profesionales, se estableció la metodología mediante la cual se llevó a cabo la propuesta para el rediseño de la red meteorológica conjunta en el área geográfica de Cundinamarca.

Una vez realizado el inventario de estaciones meteorológicas de la red, se procedió a completar y ajustar las coordenadas, nombres y categorías haciendo uso de catálogos, mapas e informes suministrados por las dos entidades, así como de la opinión de expertos. Con esta información se construyeron los mapas de ubicación de estaciones y luego, aplicando los métodos y teorías de apoyo, se trazaron los perímetros de representatividad (envolventes) para determinar, finalmente, la cobertura de la red.
Los mapas diseñados bajo la plataforma ArcGIS 9.2. En primer lugar, la georreferenciación de las estaciones (ubicación sobre los mapas) se tomó a partir de los registros de coordenadas existentes en los catálogos de las instituciones que operan la red; en segundo lugar, los límites y área de cobertura de cada uno de los puntos de observación se construyeron usando la herramienta de geo-procesamiento buffer de la interface del Arctoolbox, respetando el radio de influencia que se especificó en la metodología.

Una vez conocida la cobertura de la red pluviométrica (todas aquellas estaciones dotadas de un instrumento para medir la precipitación) y la de la red climatológica, fue posible identificar aquellas áreas donde no existen estaciones meteorológicas, donde hay sobrepoblación de estas y aquellas donde, a pesar de existir estaciones, no se cuenta con el instrumental necesario para una vigilancia adecuada de las condiciones atmosféricas.

\section{A. Condiciones observadas y ajustes a la red.}

Mediante los mapas elaborados como parte del análisis fue posible identificar las extensas áreas desprovistas de información meteorológica. En municipios como Puerto Salgar, Yacopí, La Palma, Tocaipi, Paime (norte y noroccidente de Cundinamarca), Tibirita, Machetá, Manta, Guatavita y La Calera (al oriente), Nilo (suroccidente), así como en la parte media de la cuenca del río Magdalena y la parte alta de la región del Sumapáz (municipio de Cabrera y límites rurales del Distrito Capital de Bogotá), no hay estaciones climatológicas que permitan hacer seguimiento a las condiciones atmosféricas. En estos sectores del departamento deben reubicarse algunas de las estaciones climatológicas y pluviométricas existentes y emplazar nuevos puntos de observación, con el fin de mejorar el cubrimiento de la red.

Como resultado del análisis se concluye que, para lograr una cobertura ideal de la red que opera en Cundinamarca es necesario realizar 34 cambios (entre traslados y nuevas 
instalaciones). Las estaciones que se han de trasladar tienen la siguiente distribución: tres climatológicas principales (CP), una meteorológica especial (ME) y cuatro automáticas (AU); adicionalmente, se propone la instalación de tres estaciones climatológicas principales (CP) y seis ordinarias (CO). En materia de pluviometría, la propuesta se resume al traslado de ocho pluviógrafos (PG) y seis pluviómetros (PM). Aunque el rediseño de la red meteorológica se planteó con base en el instrumental con que cuenta la red (debido al bajo presupuesto), el número limitado de estaciones activas, obliga a sugerir la instalación de tres nuevos pluviómetros (ver figura 2) [5].

Antes de instalar las estaciones meteorológicas propuestas, se deben efectuar las correspondientes visitas de campo con el fin de que los sitios de emplazamiento cumplan con los requisitos mínimos para su buen funcionamiento. Es de anotar que, los sitios seleccionados deben contar con las condiciones topográficas que permitan una buena instalación y un fácil acceso a las estaciones; además, deben estar cerca de asentamientos o poblaciones que faciliten la consecución de observadores.

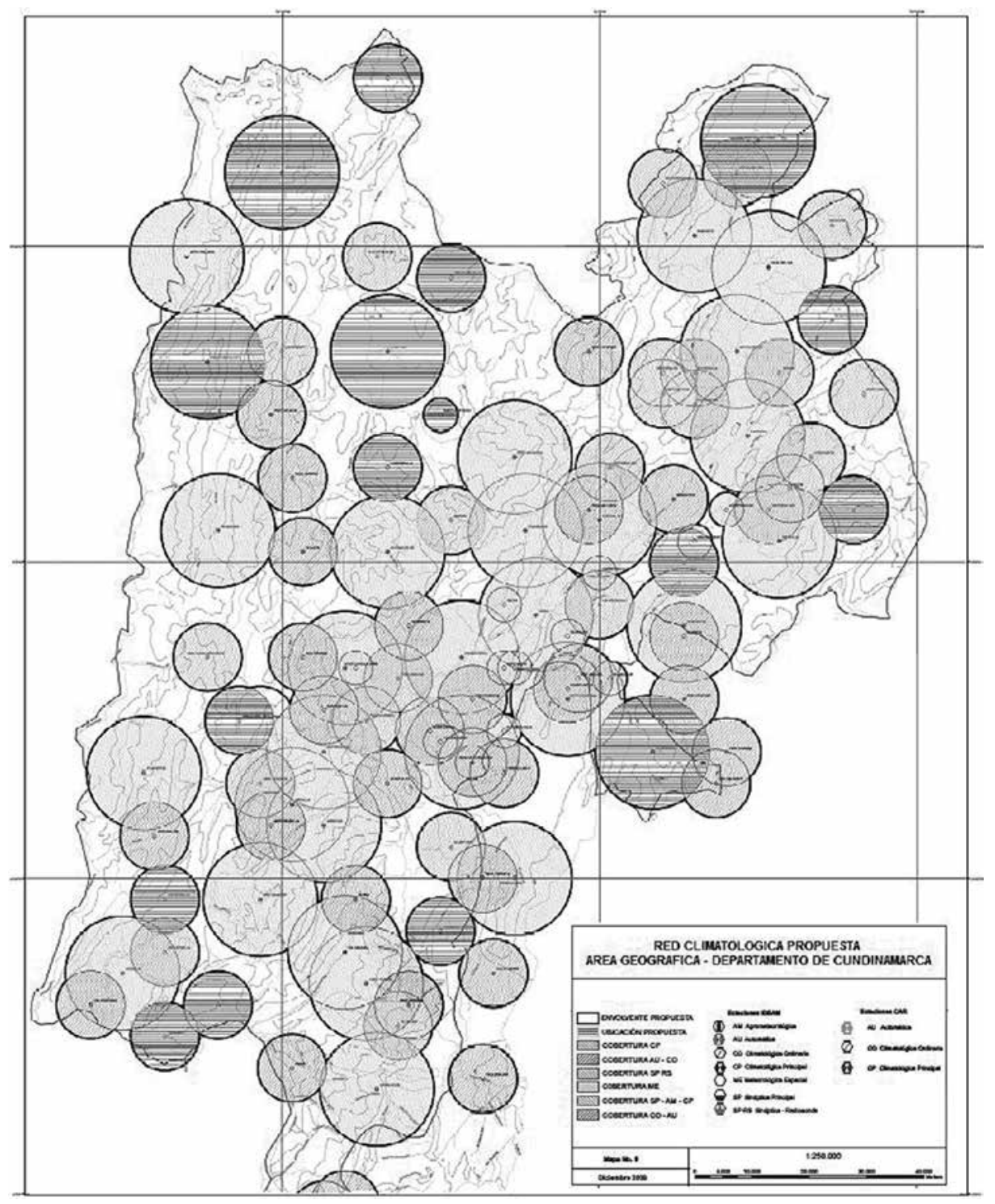

Fuente: Atlas Ambiental CAR 200

Fig. 2 Red Meteorológica de Cundinamarca. 


\section{Conclusiones}

Una vez analizadas las notas técnicas publicadas por la OMM [17] sobre diseño y emplazamiento de redes meteorológicas, elaborados los mapas de ubicación de las estaciones que conforman la red meteorológica conjunta, establecidos los radios de cobertura de acuerdo con la categoría de las estaciones y hecho el análisis preliminar de la calidad de información de la red que opera en Cundinamarca (área piloto), se llegó a las siguientes conclusiones:

La red meteorológica operada en el área geográfica de Cundinamarca no obedece a una planificación conjunta, lo que da como resultado una mala distribución de las estaciones. Esto, por consiguiente, da como resultado que extensas áreas queden sin cobertura, dificultando el seguimiento a las variables que determinan las condiciones climatológicas de la región.

Debido a la mala distribución de la red, también es posible observar exceso (alta densidad) de estaciones en algunos sectores de la jurisdicción, situación que da como resultado un mal aprovechamiento del instrumental y, como es de suponer, la duplicidad de información. Es notoria, además, la falta de estaciones meteorológicas de primer orden (CP y AM) dotadas de registradores que permitan brindar una mejor cobertura y que ofrezcan la posibilidad de servir como referentes para el monitoreo de estaciones de menor categoría como las CO, ME y PM.

Es evidente, la escases de personal capacitado para la operación de las estaciones meteorológicas convencionales, así como la dificultad en la calibración y mantenimiento de las estaciones automáticas. De otro lado, el grupo de inspectores es cada vez más reducido mientras que la necesidad de atención de la red crece, en razón a que se han venido instalando nuevos equipos tratando de mejorar la cobertura.
El emplazamiento de las estaciones automáticas se ha venido haciendo sin tener en cuenta que este tipo de instrumental requiere de la cobertura de estaciones convencionales de primer orden que permitan monitorear el comportamiento de los sensores. Por esta razón, en algunas estaciones automáticas puede estarse generando información poco confiable.

De acuerdo con las teorías sobre diseño de redes expuestas por la OMM [1], [7], regiones como la seleccionada para el estudio deben contar con una red de referencia, conformada por estaciones con buen emplazamiento, que sean representativas de las condiciones físicas del territorio, operadas por un personal de observadores capacitado, dotadas con instrumental de calidad, con una buena frecuencia de visitas técnicas, una alta posibilidad de permanencia en el lugar y con series de datos consistentes.

\section{ReCOMENDACIONES}

Analizados los resultados y conclusiones del presente estudio, el cual fue adelantado con el fin de identificar los problemas que presenta la red de estaciones meteorológicas en la región Andina colombiana, se recomienda tener en cuenta las siguientes consideraciones:

Es muy importante adelantar un proceso de identificación de aquellas estaciones de la red que cumplen con las recomendaciones técnicas de la OMM y que, por la calidad de su instrumental y su buena operación podrían garantizar el suministro de información confiable. Mediante este proceso se daría inicio a la conformación de la red de referencia.

Se considera que a partir del desarrollo de proyectos piloto en cuencas y otras unidades territoriales y de la conformación de redes de referencia y aplicando métodos estadísticos como el propuesto por Kagan, se puede llegar a tener un seguimiento ordenado y juicioso de las condiciones climáticas locales y regionales. 
Para garantizar el éxito de proyectos de diseño y/o redimensión como el presentado en este estudio es necesario, no solo contar con equipos e instrumentos de calidad, sino con una muy buena operación de las estaciones, razón por la cual se recomienda la contratación de personal, por lo menos para la operación de las estaciones escogidas como referencia. Este personal debe ser capacitado y su trabajo adecuadamente remunerado, de tal forma que se genere el compromiso necesario para la juiciosa toma de los datos y el buen manejo de la información.

Se hace necesario adelantar un proceso serio de capacitación del personal en todas y cada una de las funciones inherentes a la producción y manejo de la información meteorológica. Se recomienda formar observadores de superficie (personal clase IV-OMM), inspectores para las estaciones convencionales y automáticas de la red y verificadores de información (personal clase III-OMM) que garanticen la calidad de los datos.

Se recomienda fortalecer el procesamiento y verificación de la información meteorológica, con el fin de corregir y/o completar los datos. De esta manera, se mejorará la calidad de la información y se conseguirá una mayor consistencia en las series históricas de las estaciones que hacen parte de la red.

Como parte de las actividades propuestas para mejorar el funcionamiento de la red conjunta estudiada en este proyecto, se sugiere el desarrollo de un proceso de validación de la información generada por las estaciones automáticas, para lo cual se recomienda que las estaciones activas y las que pudieran ser instaladas en un futuro se ubiquen dentro del radio de cobertura de estaciones convencionales (CP, AM y SP), con lo que se podría realizar el monitoreo y ajuste a los sensores automáticos.

De igual manera, se considera importante unificar los procesos de generación, manejo y verificación de información de la red conjunta para, de esta manera, contar con información homogénea y de calidad en las bases de datos institucionales. Esta información deberá estar disponible para cubrir las necesidades de los investigadores, usuarios y las instituciones que componen el Servicio de Información Ambiental.

\section{RefERECias BibLIOGRAFICAS}

[1] OMM, "Guía de Instrumentos y Métodos de Observación Meteorológica". - № 8. Sexta edición. Ginebra - Suiza, 1996.

[2] Ideam, "Atlas Climatológico de Colombia", Bogotá, Imprenta Nacional, 2005, 218 p.

[3] OMM Comisión de instrumentos y métodos de observación, novena reunión, 1985.

[4] L. López, "Manual sobre manejo, análisis y verificación de información meteorológica". HIMAT, Sub-Dirección de Estudios e Investigaciones, 1988.

[5] Corporación Autónoma Regional de Cundinamarca, CAR, "Atlas Ambiental CAR", Bogotá, ed. Litografía Arco S.A., 2001, 175 p.

[6] W. Koppen. Artículo de la enciclopedia enciclopedia. us.es/index.php/Clasificación_climática_de_Köppen, 27 feb. 2012, tomado de www7.uc.cl/sw_educ/ geografia/cartografiainteractiva/.../Untitled-1.htm, abril 17 del 1013

[7] OMM“ Guía de Prácticas Hidrológicas. Adquisición y proceso de datos, análisis, predicción y otras aplicaciones, Ginebra (Suiza) 1994. No 168.

[8] Ideam, "Definición de una Red de Referencia Preliminar de Estaciones Hidro-meteorológicas con Fines de Investigación Básica y Aplicada”. Nota técnica IDEAM -003, 1996.

[9] OMM. "The planning of Meteorological Station Networks. Technical Note No. 111 by Gandín, L. S. WMO - No. 265 T. P. 149." Geneva (Switzerland). 1970.

[10] L.S. Gandín, "The Planning of Meteorological Station Networks. Technical Note No. 111. CCI Rapporteur for Climatological Networks. WMO - No. 265 T. P. 149". Geneva (Switzerland), 1970.

[11] Fopae, "Diseño de la Ampliación de la Red Hidrometeorológica de Bogotá y la Cuenca Alta del Río Tunjuelo", 2007.

[12] J. Kagan, Reflection-impulsivity: The generality and dynamics of conceptual tempo. Journal of Abnormal Psychology, Vol 71(1), Feb 19661966.

[13] OMM. "Design of Optimum Networks for Aerological Observing Stations. World Weather Watch, planning report No. 21 by Gandín, L. S. and Alaka, M. A", Geneva (Switzerland), 1967.

[14] OMM. "Guía de prácticas hidrológicas, adquisición y proceso de datos, análisis, predicción y otras aplicaciones, OMM, 1994., No. 168. 
[15] W. P. Lowry. "Compendio de apuntes de Climatología para la formación de personal meteorológico clase IV. OMM- № 237" Secretaría de la Organización, Ginebra- Suiza, 1973.

[16] Ideam-CAR, "Informe final de atividades. Convenio Inter administrativo", no 117/03-Ideam, nº 203/03-CAR

[17] OMM. "Compendio de apuntes sobre instrumentos meteorológicos para la formación del personal meteorológico de las clases III y IV', vol. I - ISBN 92-6330622-2, 1996.

[18] Instituto Humboldt, "Línea Base de la Biodiversidad en la Jurisdicción de la Corporación Autónoma Regional de Cundinamarca - CAR", Serie: Indicadores de seguimiento y evaluación de la política de biodiversidad, No. 5, Bogotá, ed. Grey Ltda., 2005, 107 p.

[19] J. Kagan (WMO): Motives and development. Abril 1972. Tomado de http://ase.tufts.edu/psychology/spacelab/pubs/Brunye_etal_Association_Accepted.pdf. 
